\title{
SESHADRI CONSTANTS ON SYMMETRIC PRODUCTS OF CURVES
}

\author{
J. Ross
}

\begin{abstract}
Let $X_{g}=C_{g}^{(2)}$ be the second symmetric product of a very general curve of genus $g$. We reduce the problem of describing the ample cone on $X_{g}$ to a problem involving the Seshadri constant of a point on $X_{g-1}$. Using this we recover a result of Ciliberto-Kouvidakis that reduces finding the ample cone of $X_{g}$ to the Nagata conjecture when $g \geq 9$. We also give new bounds on the the ample cone of $X_{g}$ when $g=5$.
\end{abstract}

\section{Introduction}

Consider the second symmetric product $C^{(2)}$ of a smooth curve $C$ of genus $g \geq 2$. This smooth surface comes with some naturally defined divisors. Given a point $p \in C$ there is the divisor $x_{p}=\{p+q: q \in C\}$ whose numerical class is independent of $p$ and will be denoted by $x$. Another divisor on $C^{(2)}$ is given by the diagonal $\Delta=\{p+p \mid p \in C\}$ whose numerical class is denoted by $\delta$.

We will be interested in describing the the intersection $N$ of the ample cone with the plane in $N^{1}\left(C^{(2)}\right)_{\mathbb{R}}$ spanned by $x$ and $\delta$. Note that when $C$ is a very general curve the classes $x$ and $\delta / 2$ generate $N^{1}\left(C^{(2)}\right)$ so in this case $N$ is the entire ample cone of $C^{(2)}$. Since $N$ is a two dimensional cone it is described by two boundary rays. The first boundary is easily given: since the diagonal $\Delta$ is an irreducible curve of negative self-intersection it spans a boundary of the effective cone, so its dual ray is one boundary. The more interesting boundary of $N$ is characterised by the quantity

$$
\tau(C)=\inf \{s>0:(s+1) x-(\delta / 2) \text { is ample }\} .
$$

There is the obvious universal bound

$$
\tau(C) \geq \sqrt{g}
$$

coming from the fact that if $(s+1) x-(\delta / 2)$ is ample then it has positive selfintersection. The following conjecture governs the ample cone of a $C^{(2)}$ when $C$ is very general.

Conjecture 1.1. If $C$ is a very general curve of genus $g \geq 4$ then $\tau(C)=\sqrt{g}$.

This conjecture asserts that for a very general curve $C$ the other boundary of $N$ has zero-self intersection, and thus the ample cone of $C^{(2)}$ is as large as possible. It is only known to hold when $g$ is a perfect square [8].

Our aim is to give a lower bound for $\tau(C)$ in terms of the Seshadri constant of a point in $D^{(2)}$ where $D$ is a smooth curve of genus $g-1$. If $X$ is a smooth surface and

Received by the editors September 5, 2006. 
$L$ is the (numerical class of) a nef $\mathbb{R}$-divisor on $X$ the Seshadri constant at a collection of distinct points $p_{1}, \ldots, p_{m} \in X$ is defined to be

$$
\epsilon\left(p_{1}, \ldots, p_{m} ; X, L\right)=\inf _{C}\left\{\frac{L . C}{\sum_{i} \operatorname{mult}_{p_{i}} C}\right\}
$$

where the infimum is over all reduced irreducible curves $C \subset X$ passing through at least one of the $p_{i}$. We will prove the following connecting Seshadri constants and the ample cone of second symmetric products of smooth curves.

Theorem 1.2. Let $D$ be a smooth curve of genus $g-1$. Suppose $a, b>0$ are such that $a / b>\tau(D)$ and for a very general point $p \in D^{(2)}$

$$
\epsilon\left(p ; D^{(2)},(a+b) x-b(\delta / 2)\right) \geq b .
$$

Then for a very general curve $C$ of genus $g$,

$$
\tau(C) \leq \frac{a}{b} .
$$

Thus Conjecture 1.1 is implied by the following conjecture about Seshadri constants:

Conjecture 1.3. Let $D$ be a very general curve of genus $g-1$ with $g \geq 5$ and $p$ be a very general point in $D^{(2)}$. Then

$$
\epsilon\left(p ; D^{(2)},(\sqrt{g}+1) x-(\delta / 2)\right)=1 .
$$

We note that this conjecture is not easy to prove as the class $L=(\sqrt{g}+1) x-(\delta / 2)$ has degree $L^{2}=1$ so the equality in (1.4) asserts that the Seshadri constant of this $\mathbb{R}$-divisor is maximal (see 2.5). There is a general lower bound due to Ein-Lazarsfeld [6] for the Seshadri constant of very general points in surfaces with respect to integral divisors but this does not extend to the case of $\mathbb{R}$-divisors. However when $g$ is a perfect square we can apply [6] to deduce that $\epsilon\left(p ; D^{(2)}, L\right)=1$ for a very general $p \in D^{(2)}$ where $D$ is a smooth curve of genus $g-1$. Thus we get another proof that if $g \geq 4$ is a perfect square then $\tau(C)=\sqrt{g}$ for a very general curve $C$ of genus $g$.

Remark 1.5. As pointed out by Lazarsfeld, it is not the case that the analogy of Conjecture 1.3 holds for all polarised surfaces $(X, L)$ such that $L$ is an ample $\mathbb{R}$-divisor with $L^{2}=1$ and $L . E \geq 1$ for all but finitely many curves $E \subset X$. For example let $X$ be an abelian surface of type $(1, d)$ with Picard number 1 generated by the ample line bundle $L^{\prime}$ and set $L=L^{\prime} / \sqrt{2 d}$. Then $L^{2}=1$ and $L . E \geq 1$ for all irreducible curves $E \subset X$. But whenever $\sqrt{2 d}$ is irrational it is known that $\epsilon\left(p, X ; L^{\prime}\right)<\sqrt{2 d}$ (in fact it is rational [2]) so $\epsilon(p ; X, L)<1$ for all $p \in X$.

The proof of Theorem 1.2 uses a degeneration of the symmetric product that arises from letting $C$ degenerate to the nodal curve $C_{0}$ obtained by gluing two points in $D$. The same degeneration allows us to compare the multipoint Seshadri constants of $C^{(2)}$ and $D^{(2)}$. We define $\epsilon_{m}(X, L)$ to be the Seshadri constant of a collection of $m$ very general points in $X$. 
Theorem 1.6. Let $D$ be a smooth curve of genus $g-1$ and fix an integer $m \geq 1$. Suppose that there are numbers $a, b>0$ with $a / b>\tau(D)$ such that

$$
\epsilon_{m+1}\left(D^{(2)},(a+b) x-b(\delta / 2)\right) \geq b .
$$

Then for a very general curve $C$ of genus $g$ the class $(a+b) x-b(\delta / 2) \in N^{1}\left(C^{(2)}\right)$ is nef and

$$
\epsilon_{m}\left(C^{(2)},(a+b) x-b(\delta / 2)\right) \geq b .
$$

A more concise way to state this theorem is to let $\epsilon_{m, g}(s)$ be the Seshadri constant of $m$ very general points in $C^{(2)}$ with respect to the class $(s+1) x-\delta / 2$, where $C$ is a very general curve of genus $g \geq 0$. Then

$$
\epsilon_{m, g}(s) \geq \epsilon_{m+1, g-1}(s),
$$

where this is to be interpreted as holding for all $s, g$ such that the right hand makes sense.

As the second symmetric product of $\mathbb{P}^{1}$ is $\mathbb{P}^{2}$, induction on $g$ yields:

Corollary 1.7 (Ciliberto-Kouvidakis [4]). Let $C$ be a very general curve of genus $g \geq 1$. Then

$$
\tau(C) \leq \frac{1}{\epsilon_{g}\left(\mathbb{P}^{2}, \mathcal{O}_{\mathbb{P}^{2}}(1)\right)} .
$$

As is well known, one formulation of the Nagata conjecture states that if $g \geq 9$ then the Seshadri constant of $g \geq 9$ very general points in $\mathbb{P}^{2}$ is maximal:

Conjecture 1.8 (Nagata Conjecture). If $g \geq 9$ then

$$
\epsilon_{g}\left(\mathbb{P}^{2}, \mathcal{O}_{\mathbb{P}^{2}}(1)\right)=\frac{1}{\sqrt{g}} .
$$

Thus, as proved in [4], the Nagata conjecture yields the ample cone of $C^{(2)}$ for a very general curve of genus $g \geq 9$. Currently the Nagata conjecture is only proved when $g$ is a perfect square. For other $g$ there are several bounds on $\epsilon_{g}\left(\mathbb{P}^{2}, \mathcal{O}_{\mathbb{P}^{2}(1)}\right)$ (e.g. $[7,13,14,15])$. For instance using (1.7) and results from [13] we get that for a very general curve of genus $g \geq 10$,

$$
\tau(C) \leq \frac{\sqrt{g}}{\sqrt{1-\frac{1}{8 g}}} .
$$

When $g$ is not a perfect square this improves on the bound $\tau(C) \leq \frac{g}{[\sqrt{g}]}$ from [8].

Remark 1.10. From the discussion above there are implications

$$
\begin{aligned}
& \begin{array}{l}
\text { Conjecture } 1.8 \\
\text { (Nagata conjecture) }
\end{array}
\end{aligned} \Rightarrow \begin{aligned}
& \text { Conjecture 1.3 } \\
& \text { (Seshadri constants) }
\end{aligned} \Rightarrow \begin{aligned}
& \text { Conjecture 1.1 } \\
& \text { (Ample cone of } \left.C^{(2)}\right)
\end{aligned}
$$

so Conjecture 1.3 concerning Seshadri constants sits between the Nagata conjecture and the conjecture governing the ample cone of a general $C^{(2)}$. It is possible that Conjecture 1.3 is easier than the full Nagata conjecture but there is, of course, currently no proof of this. 
Remark 1.11. The degeneration we use in the proofs of the above theorems is related to the Franchetta degeneration used in [4] which arises from degenerating $C$ to a rational nodal curve. The main difference is that here we consider the case that $C$ develops one node at a time. Moreover rather than using a degeneration of $C^{(2)}$ we find it easier to use a degeneration of $C \times C$ and only consider divisors that are invariant under permuting the factors. A related degeneration of of $C^{(2)}$ coming from letting $C$ develop cusps is described in [12].

The values of $\tau(C)$ are known for a very general curve of genus $g \leq 4$ (see Section 2.2 ) and from the discussion above the Nagata conjecture governs the case that $g \geq 9$. There appears to be little known in the intermediate range $5 \leq g \leq 8$ and one would imagine that one of the two extreme cases holds (namely that either $\tau(C)=$ $\epsilon_{g}\left(\mathbb{P}^{2}, \mathcal{O}_{\mathbb{P}^{2}}(1)\right)^{-1}$ or $\tau(C)=\sqrt{g}$ for a very general curve $C$ of genus $\left.5 \leq g \leq 8\right)$. In Section 4 we apply Theorem 1.2 to show that the first case does not hold when $g=5$; more precisely we show that if $C$ is a very general curve of genus 5 then $\tau(C) \leq 16 / 7$ which gives

$$
2.236 \simeq \sqrt{5} \leq \tau(C) \leq 16 / 7 \simeq 2.286<\epsilon_{5}\left(\mathbb{P}^{2}, \mathcal{O}_{\mathbb{P}^{2}}\right)^{-1}=2.5 .
$$

To achieve this we use the techniques of Ein-Lazarsfeld [6] to get lower bounds of Seshadri constants of points in $D^{(2)}$ where $D$ is a curve of genus 4 . This bound is stronger than that obtained from Corollary 1.7 as $\epsilon_{5}\left(\mathbb{P}^{2}, \mathcal{O}_{\mathbb{P}^{2}}(1)\right)=2 / 5[13]$. The number $16 / 7$ is not expected to be optimal, but it is, as far as I am aware, the best that is currently known.

Notation and conventions: We work throughout over $\mathbb{C}$. The Néron-Severi space of divisors (resp. $\mathbb{R}$-divisors) on a variety $V$ modulo numerical equivalence is denoted $N^{1}(V)$ (resp. $N^{1}(V)_{\mathbb{R}}$ ). An $\mathbb{R}$-divisor $L$ on a variety $V$ is ample (resp. nef) if it is a formal sum $\sum_{i=1}^{r} a_{i} D_{i}$ of ample (resp. nef) divisors where the $a_{i} \in \mathbb{R}$ are positive (resp. non-negative). Equivalently $D \in N^{1}(V)_{\mathbb{R}}$ is nef if and only if it has non-negative self intersection with every irreducible curve $C \subset V$.

We say that $p \in V$ is a very general point if there is a countable collection of proper subvarieties $\left(V_{n}\right)_{n \geq 1}$ of $V$ such that $p$ is not contained in the union $\bigcup_{n \geq 1} V_{n}$. A collection $p_{1}, \ldots, p_{m}$ of points in $V$ is very general if $\left(p_{1}, \ldots, p_{m}\right) \in V^{\times m}$ is very general. By a very general curve we mean a smooth curve whose corresponding point in the moduli space $M_{g}$ is very general.

\section{Preliminaries}

2.1. Divisors the second symmetric product. Let $C$ be a smooth curve of genus $g \geq 0$. The product $C \times C$ has a natural involution and the second symmetric product is the quotient $\sigma_{C}: C \times C \rightarrow C^{(2)}$ which is a smooth surface. We denote the image of a point $(p, q) \in C \times C$ by $p+q$. In $N^{1}\left(C^{(2)}\right)$ we have the classes $x$ and $\delta$ as defined in the introduction. (Of course these classes really depend on $C$ but this will always be clear from context.) It is well known that when $C$ is a very general curve then $N^{1}\left(C^{(2)}\right)$ is spanned by $x$ and $\delta / 2([1]$ p.359) and when $g \geq 1$ they are independent. When $g=0, C^{(2)}=\mathbb{P}^{2}$ and both $x$ and $(\delta / 2)$ are the class of the hyperplane.

The intersection of these classes is given by $x^{2}=1, \delta^{2}=4-4 g$, and $x . \delta=2$, so

$$
((n+\gamma) x-\gamma(\delta / 2)) \cdot\left(\left(n^{\prime}+\gamma^{\prime}\right) x-\gamma^{\prime}(\delta / 2)\right)=n n^{\prime}-\gamma \gamma^{\prime} g .
$$


Notice that if $(n+\gamma) x-\gamma(\delta / 2)$ is effective then intersecting with the ample class $x$ implies $n>0$.

As in the introduction define

$$
\begin{aligned}
\tau(C) & =\inf \{s>0:(s+1) x-(\delta / 2) \text { is ample }\} \\
& =\min \{s \geq 0:(s+1) x-(\delta / 2) \text { is nef }\} .
\end{aligned}
$$

Since $(\tau(C)+1) x-(\delta / 2)$ is nef, it has non-negative self-intersection which yields

$$
\tau(C) \geq \sqrt{g} .
$$

Now as is standard in such situations, the function $\tau(C)$ is semicontinuous with respect to $C$ :

Lemma 2.1. Let $X \rightarrow T$ be a flat family of smooth curves over an irreducible base $T$ and for $t \in T$ denote the fibre by $C_{t}$. If $t_{0} \in T$ is fixed then

$$
\tau\left(C_{t}\right) \leq \tau\left(C_{t_{0}}\right) \text { for very general } t \in T .
$$

Proof. Let $Y \rightarrow T$ be the relative second symmetric product of $X$, and denote the fibre of $Y$ over $t$ by $Y_{t}$. Let $\mathcal{D} \subset Y$ be the diagonal and pick a divisor $D$ on $Y$ which has class $x$ on a each fibre $Y_{t}$. If $\tau=\tau\left(C_{t_{0}}\right)$ then by hypothesis $F=(\tau+1) D-(\mathcal{D} / 2)$ restricts to a nef divisor on $Y_{t_{0}}$. Hence for very general $t$ the restriction if $F$ to $Y_{t}$ is nef ([10] 1.4.14) which implies $\tau\left(C_{t}\right) \leq \tau$.

In particular by applying this to a complete family of smooth curves we see that if $\tau(C) \leq \tau_{0}$ for some smooth curve $C$ of genus $g$, then the same bound holds for a very general curve of genus $g$. Moreover if $C$ is a very general curve then $\tau(C)$ is independent of the actual curve chosen.

A geometric interpretation of Conjecture 1.1 can be given in terms of the existence of "exceptional" curves in $C^{(2)}$ :

Lemma 2.2. Let $C$ be a smooth curve of genus $g \geq 2$.

(1) If $\tau(C)>\sqrt{g}$ then there exists a reduced irreducible curve $D \subset C^{(2)}$ with numerical class $(n+\gamma) x-\gamma(\delta / 2)+\sigma$ where $\sigma \cdot x=\sigma . \delta=0$ such that $\tau(C)=\frac{\gamma g}{n}$.

(2) If $C$ is a very general curve then $\tau(C)=\sqrt{g}$ if and only if $\Delta$ is the only reduced irreducible curve in $C^{(2)}$ with negative self-intersection. Moreover if there does exist another such curve of negative self intersection then it is unique.

Proof. Suppose $\tau=\tau(C)>\sqrt{g}$. Then the $\mathbb{R}$-divisor

$$
F=(\tau+1) x-(\delta / 2)
$$

has positive self-intersection and, by definition of $\tau$, is nef but not ample. Thus by the Nakai criterion for real divisors [3] there is a reduced irreducible curve $D \subset C^{(2)}$ with $D . F=0$. We can write the numerical class of $D$ as $(n+\gamma) x-\gamma(\delta / 2)+\sigma$ where $\sigma$ is a class orthogonal to $x$ and $\delta$ so $D . F=0$ implies $\tau=\frac{\gamma g}{n}$. We note that since $n>0$ this implies $\gamma>0$.

Now suppose $C$ is very general. Then the effective cone of $C^{(2)}$ is spanned by $x$ and $\delta / 2$. Thus if $\tau=\tau(C)>\sqrt{g}$ the curve $D$ above has class $(n+\gamma) x-\gamma(\delta / 2)$ (i.e. 
$\sigma=0)$. Hence $D^{2}=n^{2}-g \gamma^{2}=\frac{n^{2}}{g}\left(g-\tau^{2}\right)<0$ so $D$ has negative self intersection and clearly $D \neq \Delta$ as $\gamma>0$.

Before proving the converse we deal with uniqueness. To this end suppose that $(n+\gamma) x-\gamma(\delta / 2)$ and $\left(n^{\prime}+\gamma^{\prime}\right) x-\gamma^{\prime}(\delta / 2)$ are classes of distinct reduced irreducible curves of negative self intersection. Then $n^{2}-\gamma^{2} g<0, n^{\prime 2}-\gamma^{\prime 2}<0$ and $n n^{\prime}-\gamma \gamma^{\prime} g \geq 0$ which implies $\gamma_{1}$ and $\gamma_{2}$ have opposite sign. Hence any irreducible curve $D \neq \Delta$ in $C^{(2)}$ with $D^{2}<0$ must have numerical class $(n+\gamma) x-\gamma(\delta / 2)$ with $\gamma>0$, and if it exists it is unique. Thus if there exists a reduced irreducible curve $D \neq \Delta$ with negative self intersection it has numerical class $(n+\gamma) x-\gamma(\delta / 2)$ with $\gamma>0$ and $D^{2}=n^{2}-g \gamma^{2}<0$. As $F$ is nef we know $0 \leq F . D=\tau n-g \gamma$ which implies $\tau \geq \frac{g \gamma}{n}>\sqrt{g}$.

Before proceeding with the main results of this paper we digress to discuss a finiteness result concerning the possible values of $\tau(C)$ as $C$ ranges over all curves of fixed genus $g \geq 2$.

Proposition 2.3. Fix a real number $\alpha>\sqrt{g}$. Then

$$
\{\tau \geq \alpha: \tau=\tau(C) \text { for some smooth curve } C \text { of genus } g\}
$$

is a finite set. Equivalently the only possible accumulation point of the set given by $\{\tau(C): C$ a smooth curve of genus $g\}$ is $\sqrt{g}$.

Proof. Fix a number $s \in(\sqrt{g}, \alpha) \cap \mathbb{Q}$. We first prove that there exists an integer $k$ such that for any smooth curve $C$ of genus $g$ the divisor $k[(s+1) x-(\delta / 2)]$ on $C^{(2)}$ is effective.

To this end let $C$ be any smooth curve of genus $g$ and fix a $\mathbb{Q}$-divisor $F$ on $C^{(2)}$ whose numerical class is $(s+1) x-(\delta / 2)$. We note that the canonical class of $C^{(2)}$ is $K=(2 g-2) x-(\delta / 2)$ ([9] Prop. 2.6). Thus if $k \in \mathbb{N}$ is sufficiently large (with $k s \in \mathbb{N})$ then $x .(K-k F)<0$. So by Serre duality $h^{2}(\mathcal{O}(k F))=h^{0}(\mathcal{O}(K-k F))=0$ as $x$ is ample. Hence for such $k$,

$$
h^{0}(\mathcal{O}(k F)) \geq h^{0}(\mathcal{O}(k F))-h^{1}(\mathcal{O}(k F))=\chi(k F)=p(k)
$$

where by the Riemann-Roch theorem $p(k)$ is a polynomial whose coefficients depend only on $s$ and $g$ (and not on the specific curve $C$ or choice of $F$ ). The leading order coefficient of $p(k)$ is $F^{2} / 2=\left(s^{2}-g\right) / 2>0$ so there exists a $k$ (independent of $C$ ) such that $h^{0}(\mathcal{O}(k F))>0$ as claimed.

Now suppose $C$ is chosen so that $\tau(C) \geq \alpha$. By Lemma 2.2(a) there exists a reduced irreducible curve $D \subset C^{(2)}$ with numerical class $(n+\gamma) x-\gamma(\delta / 2)+\sigma$ where $\sigma . x=\sigma . \delta=0$ and $\tau(C)=\frac{g \gamma}{n}$. With $k, F$ as above there is a divisor $E \subset|k F|$. But as $s<\alpha \leq \tau(C)$ we have $D . F=(n s-\gamma g)<0$ so $D \subset E$. Since $D$ is reduced this implies that $n=x . D \leq x . E=k s$. Thus letting $N:=k s$ we have $n \leq N$. To complete the proof note that the divisor $G=(g-1) x+(\delta / 2)$ is always nef as it is the pullback of the theta divisor under the Abel-Jacobi map $u: C^{(2)} \rightarrow \operatorname{Jac}(C)$. Hence $D \subset E$ also implies $D . G \leq E$. $G$ which yields $g n+\gamma g \leq k(g s+g)$ so $\gamma \leq k(s+1)=: M$. Thus $\tau(C)$ lies in the set

$$
\left\{\tau: \tau=\frac{g \gamma}{n} \text { with } n, \gamma \in \mathbb{N} \text { and } n \leq N, \gamma \leq M\right\}
$$

which is finite. 
Remark 2.4. A similar finiteness result for Seshadri constants in families of surfaces can be found in [11]. It would be interesting to know if the finiteness from Proposition 2.3 still holds when $\alpha=\sqrt{g}$.

2.2. The case of low genus. For low genus it is possible to describe the intersection of the ample cone with the plane spanned by $x$ and $\delta$ by finding explicit irreducible curves of negative self intersection. For details see [4, 8] (or [10] Section 1.5.B).

- $g=0$ : Here $C^{(2)}=\mathbb{P}^{2}$ and $(s+1) x-(\delta / 2)=s h$ where $h$ is the class of the hyperplane, so trivially $\tau\left(\mathbb{P}^{1}\right)=0$.

- $g=1$ : In this case it is well known that if $C$ is a very general genus 1 curve then the closure of the effective cone of $C^{(2)}$ is the nef cone. It is a closed circular cone described by the equations $\alpha^{2} \geq 0, \alpha . h \geq 0$ where $h$ is an ample class ([10] Lemma 1.5.4). Thus $\tau(C)=1$.

- $g=2$ : Any curve $C$ of genus 2 is hyperelliptic. Using the $g_{1}^{2}$ one can produce an irreducible curve in $C^{(2)}$ of negative self intersection whose class is $2 x-$ $(\delta / 2)$, and thus $\tau(C)=2$.

- $g=3$ : If $C$ is a very general curve of genus 2 then it is possible to construct an irreducible curve in $C^{(2)}$ whose class is $16 x-6(\delta / 2)$ and thus has selfintersection -8 $[4,8]$. Using this one deduces that $\tau(C)=9 / 5$.

- $g=4$ : If $C$ is a very general curve of genus 4 then $\tau(C)=2$. In fact any such curve admits two $g_{1}^{3}$, and the associated $\Gamma_{3}$ is an irreducible curve whose class is $3 x-(\delta / 2)$ spans one boundary of the effective cone. (This can also be obtained from Corollary 1.7).

2.3. Seshadri constants. We record some basic definitions and properties of Seshadri constant and refer the reader to $[2,10]$ for a comprehensive treatment. Let $X$ be a smooth variety of dimension $n$ and $L$ be a nef numerical class in $N^{1}(X)_{\mathbb{R}}$. If $p_{1}, \ldots, p_{m}$ are points in $X$ define

$$
\epsilon\left(p_{1}, \ldots, p_{m} ; X, L\right)=\inf _{C}\left\{\frac{L . C}{\sum_{i=1}^{r} \operatorname{mult}_{p_{i}} C}\right\},
$$

where the infimum is over all reduced irreducible curves $C$ in $X$ that pass through at least one of the $p_{i}$. Equivalently if $\pi: B \rightarrow X$ is the blowup of $X$ at these points with exceptional divisor $E$ then

$$
\epsilon\left(p_{1}, \ldots, p_{m} ; X, L\right)=\max \left\{s \geq 0: \pi^{*} L-s E \text { is nef }\right\} .
$$

By a standard semicontinuity argument similar to (2.1) the Seshadri constant of $m$ very general points does not depend on the actual points chosen. Thus we can set

$$
\epsilon_{m}(X, L)=\epsilon\left(p_{1}, \ldots, p_{m} ; X, L\right) \text { where } p_{1}, \ldots, p_{m} \text { are in very general position. }
$$

Notice that if $\pi^{*} L-c E$ is nef then $\left(\pi^{*} L-c E\right)^{n} \geq 0$ which implies

$$
\epsilon\left(p_{1}, \ldots, p_{m} ; X, L\right) \leq \sqrt[n]{\frac{L^{n}}{m}}
$$

It is an interesting and difficult problem to get non-trivial bounds for Seshadri constants in general $[5,6]$ or even to calculate them in examples (see [10] and the reference therein).

We will make use of the following simple lemma which says that Seshadri constants of a collection of points in $C^{(2)}$ can be calculated by looking at their preimage in $C^{\times 2}$. 
Lemma 2.6. Let $D$ be a smooth curve and $\sigma_{D}: D^{\times 2} \rightarrow D^{(2)}$ be the quotient map. Let $p_{1}, \ldots, p_{m}$ be general points in $D^{(2)}$ and suppose for each $i$ that $\sigma_{D}^{-1}\left(p_{i}\right)=\left\{q_{i}^{1}, q_{i}^{2}\right\}$. Then for any nef class $L \in N^{1}\left(D^{(2)}\right)_{\mathbb{R}}$,

$$
\epsilon\left(p_{1}, \ldots, p_{m} ; D^{(2)}, L\right)=\epsilon\left(q_{1}^{1}, q_{1}^{2}, \ldots, q_{m}^{1}, q_{m}^{2} ; D^{\times 2}, \sigma_{D}^{*} L\right) .
$$

Proof. Let $p_{X}: X \rightarrow D^{(2)}$ be the blowup of $D^{(2)}$ at $p_{1}, \ldots, p_{m}$ with exceptional divisor $E$, and $p_{Y}: Y \rightarrow D^{\times 2}$ be the blowup of $D^{\times 2}$ at $q_{1}^{1}, q_{2}^{2}, \ldots, q_{m}^{1}, q_{m}^{2}$ with exceptional divisor $F$. Then under the induced map $\tilde{\sigma}_{D}: Y \rightarrow X$ that lifts $\sigma_{D}$ we have for any $c>0$ that $\tilde{\sigma}_{D}^{*}\left(p_{X}^{*} L-c E\right)=p_{Y}^{*} L-c F$. Since $\tilde{\sigma}_{D}$ is surjective this implies $p_{X}^{*} L-c E$ is nef if and only if $p_{Y}^{*} L-c F$ is nef, which proves the lemma.

\section{Proofs}

Let $\mathcal{C} \rightarrow T$ be a family of smooth curves of genus $g$ over a disc $T$ that develops a node. By this we mean that the family is proper and flat, the fibre $C_{t}$ over $t \in T$ is a smooth curve for $t \neq 0$ and that the fibre $C_{0}$ over $0 \in T$ is an irreducible curve that has a single node. We assume further that $\mathcal{C}$ has a smooth total space. Taking the relative second symmetric product of $\mathcal{C}$ gives a degeneration of $C^{(2)}$ which when suitably blown up is essentially the Franchetta degeneration used in [4]. Instead of using this we find it easier to consider the fibred product $\mathcal{C} \times{ }_{T} \mathcal{C}$ (and thus a degeneration of $C \times C$ ) and only deal with divisors that are invariant under permuting the factors.

To this end suppose $p$ is the node in $C_{0}$ and let $\mathcal{Y} \rightarrow \mathcal{C} \times{ }_{T} \mathcal{C}$ be the blowup of $\mathcal{C} \times{ }_{T} \mathcal{C}$ at $(p, p)$ with exceptional divisor $E$. One can easily check by working in local analytic coordinates that $\mathcal{Y}$ has smooth total space. Clearly the fibre of $\mathcal{Y}$ over $t \neq 0$ is $\mathcal{Y}_{t}=C_{t} \times C_{t}$ and the next two lemmas describe the central fibre of $\mathcal{Y}$. Denote the normalisation of $C_{0}$ by $D$ and let $q, r \in D$ be the preimage of the node $p \in C_{0}$.

\section{Lemma 3.1.}

(1) The central fibre $\mathcal{Y}_{0}$ has two irreducible components namely the exceptional divisor $E$ which is isomorphic to $\mathbb{P}^{1} \times \mathbb{P}^{1}$ and another we denote by $F$.

(2) The normalisation $\tilde{F}$ of $F$ is the blowup $\pi: \tilde{F} \rightarrow D \times D$ at the four points $(q, q),(q, r),(r, q),(r, r)$ making the natural diagram

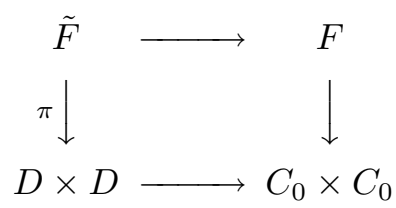

commute. We denote the exceptional curve in $\tilde{F}$ that sits over the point $(s, t)$ by $\tilde{e}_{s t}$ and the corresponding curve in $F$ by $e_{s t}$.

(3) The two components of $\mathcal{Y}_{0}$ are glued along the four rational curves $\left\{e_{q q}, e_{r r}\right\}$ and $\left\{e_{q r}, e_{r q}\right\}$ in $F$. Each set consists of a pair of lines in one of the two rulings of $E$.

Proof. In local analytic coordinates around the node $p \in C_{0}$ the family $\mathcal{C}$ has the form $x y=t$ in $\mathbb{C}^{2} \times T$ where $t$ is the parameter on $T$. Hence locally $\mathcal{C} \times_{T} \mathcal{C}$ is given by $x_{1} y_{1}=x_{2} y_{2}=t$ in $\mathbb{C}^{4} \times T$. Thus a local model for $\mathcal{Y}$ is given by the proper 
transform in the blowup $B \rightarrow \mathbb{C}^{4} \times T$ at the origin. On the exceptional $\mathbb{P}^{4}$ in $B$ we pick coordinates $\lambda_{1}, \lambda_{2}, \mu_{1}, \mu_{2}, \sigma$ such that for $t \neq 0$

$$
\frac{\lambda_{i}}{x_{i}}=\frac{\mu_{j}}{y_{j}}=\frac{\sigma}{t} \quad i, j=1,2 .
$$

Then $E$ is the intersection of $\mathcal{Y}$ with $\mathbb{P}^{4}$ and is given by

$$
\lambda_{1} \mu_{1}=\lambda_{2} \mu_{2} \text { and } \sigma=0
$$

which is a quadric hypersurface in $\mathbb{P}^{3}$, and thus $E \simeq \mathbb{P}^{1} \times \mathbb{P}^{1}$.

Now let $U$ and $V$ be the two components of the normalisation of $x y=t$ corresponding to $x=0$ and $y=0$ respectively. Then locally the other components of $\mathcal{Y}$ are the proper transform of $U \times U, U \times V, V \times U$ and $V \times V$ given by $x_{1}=x_{2}=0$, $x_{1}=y_{2}=0, y_{1}=x_{2}=0$ and $y_{1}=y_{2}=0$ respectively. These are glued along normal crossing curves and the normalisation $\tilde{F}$ is obtained by pulling them apart. Thus $\tilde{F}$ is the blowup of $D \times D$ in the four points as claimed.

Now the proper transform of $U \times U$ is the blowup at the point $(p, p)$ and meets $E$ in the line given by $\lambda_{1}=\lambda_{2}=0$. Similarly $V \times V$ meets $E$ in the line $\mu_{1}=\mu_{2}$ which is easily seen to be in the same ruling. A completely analogous analysis applies to $U \times V$ and $V \times U$.

For simplicity denote the numerical class of the curves $\tilde{e}_{s t}$ by the same letter. Then

$$
N^{1}(\tilde{F})=\pi^{*} N^{1}(D \times D) \oplus \mathbb{Z}\left[\tilde{e}_{q q}, \tilde{e}_{r r}, \tilde{e}_{q r}, \tilde{e}_{r q}\right] .
$$

Moreover $N^{1}(E)$ is a free group of rank 2 with two generators $\alpha$ and $\beta$. We declare that $\alpha$ is the class of the curve $e_{q q}$ (equivalently of $e_{r r}$ ) inside $E$ and $\beta$ is the class of $e_{q r}$ (equivalently of $e_{r q}$ ).

Consider now the proper transform $\mathcal{D} \subset \mathcal{Y}$ of the diagonal in $\mathcal{C} \times_{T} \mathcal{C}$.

Lemma 3.2. The restriction of $\mathcal{D}$ to $E$ has class $\alpha \in N^{1}(E)$. The pullback of $\left.\mathcal{D}\right|_{F}$ to $\tilde{F}$ is the proper transform of the diagonal $\Delta_{D} \subset D \times D$ and thus has class $\pi^{*} \Delta_{D}-\tilde{e}_{q q}-\tilde{e}_{r r} \in N^{1}(\tilde{F})$.

Proof. We continue to use the local coordinates introduced in the proof of Lemma 3.1. For $t \neq 0$ the diagonal is given by $x_{1}=y_{1}$ and $x_{2}=y_{2}$ and thus meets $E$ in the line $\lambda_{1}=\mu_{1}$ and $\lambda_{2}=\mu_{2}$. As is easily checked this line has class $\alpha$.

Now clearly the proper transform of $\left.\mathcal{D}\right|_{U \times U}$ is, locally, the proper transform of the diagonal $y_{1}=y_{2}$ in $U \times U$ and similarly for $V \times V$. Moreover for $\Delta_{D}$ is disjoint from $U \times V$ and $V \times U$ for $t \neq 0$ and thus the pullback of $\left.\mathcal{D}\right|_{F}$ to $\tilde{F}$ has the numerical class as claimed.

With these preliminaries we are ready to give the proofs of the theorems stated in the introduction.

Proof of Theorem 1.2. By hypothesis there is a smooth curve $D$ of genus $g-1$ and distinct points $q, r \in D$ such that

$$
\epsilon\left(q+r ; D^{(2)},(a+b) x-b(\delta / 2)\right) \geq b .
$$

By gluing $q$ and $r$ we get a curve $C_{0}$ with a single node $p$ whose arithmetic genus is $g$ and whose normalisation is $D$. Let $\mathcal{C} \rightarrow T$ be a proper flat family of curves of genus $g$ which has a smooth total space, smooth general fibre and whose central 
fibre is $C_{0}$. Let $\mathcal{Y} \rightarrow \mathcal{C} \times_{T} \mathcal{C}$ be the blowup at $(p, p)$. We will use the notation introduced in Lemmas 3.1 and 3.2 so the central fibre $\mathcal{Y}_{0}$ has two components $E$ and $F$, and the normalisation $\tilde{F}$ of $F$ is the blowup $\pi: \tilde{F} \rightarrow D \times D$ at the four points $(q, q),(q, r),(r, q),(r, r)$.

Fix a line bundle $L$ on $\mathcal{C} / \mathcal{C}_{0}$ that has degree 1 on each of the fibres. As $\mathcal{C}$ is assumed to be smooth and $\mathcal{C}_{0}$ is irreducible, $L$ extends uniquely to a line bundle $L^{\prime}$ on all of $\mathcal{C}$. Take a meromorphic section of $L^{\prime}$ whose support does not contain the node $p$ of $C_{0}$. By shrinking $T$ if necessary we may assume furthermore that the support of $s$ does not contain any fibre $\mathcal{C}_{t}$, and we write this support as $\sum_{i} a_{i} D_{i}$ for some divisors $D_{i} \subset \mathcal{C}$ that do not contain $p$. For each $i$ define a divisor on $\mathcal{Y}$ by

$$
G_{i}=\left\{(u, v) \in \mathcal{Y}_{t} \text { for some } t \text { and either } u \in D_{i} \text { or } v \in D_{i}\right\},
$$

and set

$$
G=\sum_{i} a_{i} G_{i}
$$

Clearly $G$ is invariant under the natural involution on $\mathcal{Y}$. In fact for for all $t \neq 0$ the numerical class of $G{\mid \mathcal{Y}_{t}}_{\text {is }}$ the pullback of $x$ under $\sigma_{C_{t}}: C_{t} \times C_{t} \rightarrow C_{t}^{(2)}$. Moreover $G$ is trivial along $E$ and and the numerical class of the pullback of $\left.G\right|_{F}$ to $\tilde{F}$ has class $\pi^{*} \sigma_{D}^{*} x$.

Let $\mathcal{D} \subset \mathcal{Y}$ be the proper transform of the diagonal in $\mathcal{C} \times{ }_{T} \mathcal{C}$ as in Lemma 3.2 and define an $\mathbb{R}$-divisor on $\mathcal{Y}$ by

$$
H=(a+b) G-b(\mathcal{D}+E) .
$$

We claim that $\left.H\right|_{\mathcal{Y}_{0}}$ is nef. To see this note first from $(3.1,3.2)$ that $\left.E\right|_{E}$ has class $-(2 \alpha+2 \beta)$ and $\left.\mathcal{D}\right|_{E}$ has class $\alpha$. Thus $\left.H\right|_{E}$ has class $b(\alpha+2 \beta)$ which is clearly nef as both $\alpha$ and $\beta$ are nef and $b>0$. To show that $\left.H\right|_{F}$ is nef consider its pullback to $\tilde{F}$ which using $(3.1,3.2)$ has numerical class

$$
\begin{aligned}
(a+b) \pi^{*} \sigma_{D}^{*} x-b\left(\pi^{*} \Delta_{D}-\tilde{e}_{q q}-\tilde{e}_{r r}\right)-b\left(\tilde{e}_{q q}+\tilde{e}_{r r}+\tilde{e}_{r q}+\tilde{e}_{r q}\right) & \\
= & \pi^{*} \sigma_{D}^{*}((a+b) x-b(\delta / 2))-b\left(\tilde{e}_{r q}+\tilde{e}_{r q}\right)
\end{aligned}
$$

since $\sigma_{D}^{*}(\delta / 2)=\Delta_{D}$. Now $\sigma_{D}^{-1}(q+r)=\{(q, r),(r, q)\}$ so (3.3) and Lemma 2.6 imply

$$
\epsilon\left((q, r),(r, q) ; D \times D, \sigma_{D}^{*}((a+b) x-b(\delta / 2))\right) \geq b .
$$

But by (3.5) this means exactly that the pullback of $\left.H\right|_{F}$ to $\tilde{F}$ is nef and thus $\left.H\right|_{F}$ is nef as well.

Hence $\left.H\right|_{\mathcal{Y}_{0}}$ is nef and by semicontinuity so is $\left.H\right|_{\mathcal{Y}_{t}}$ for very general $t$. But $H_{\mathcal{Y}_{t}}$ has class $\sigma_{C_{t}}^{*}((a+b) x-b(\delta / 2))$ so $(a+b) x-(\delta / 2) \in N^{1}\left(C_{t}^{(2)}\right)$ is nef for very general $t$ which proves that $\tau\left(C_{t}\right) \leq \frac{a}{b}$. By $(2.1)$ the same inequality holds for any very general curve of genus $g$.

Proof of Theorem 1.6. The fact that $(a+b) x-b(\delta / 2) \in N^{1}\left(D^{(2)}\right)$ is nef comes from Theorem 1.2 since $\epsilon_{r}(\cdot) \leq \epsilon_{1}(\cdot)$. Essentially the result we want comes from the degeneration $\mathcal{Y}$ described in the proof of Theorem 1.2 and semicontinuity of Seshadri constants in families.

To describe this more explicitly we continue the notation from the above proof. Pick $m$ sections $s_{1}, \ldots, s_{m}$ of $\mathcal{C} \times_{T} \mathcal{C} \rightarrow T$ that meet $C_{0} \times C_{0}$ at $m$ very general points (so in particular these points are not equal to $(p, p)$ ). Let $V_{i}$ be the image of $s_{i}$ and 
$V_{i}^{\prime}$ be the image of $V_{i}$ under the involution. We denote the proper transform of $V_{i}$ and $V_{i}^{\prime}$ in $\mathcal{Y}$ by $W_{i}$ and $W_{i}^{\prime}$ and let $W=\bigcup_{i} W_{i} \cup W_{i}^{\prime}$. By shrinking $T$ if necessary we may assume that $W$ meets each fibre $\mathcal{Y}_{t}=C_{t} \times C_{t}$ at a collection of $2 m$ distinct points. Note that in the central fibre $\mathcal{Y}_{0}$ these points are all in the component $F$.

Now let $\pi: \mathcal{Y}^{\prime} \rightarrow \mathcal{Y}$ be the blowup along $W$ with exceptional divisor $E^{\prime}$. The central fibre of $\mathcal{Y}^{\prime}$ has components $E$ and $F^{\prime}$ where the normalisation $\tilde{F}^{\prime}$ of $F^{\prime}$ is the blowup of $\tilde{F}$ at $m$ very general points and their image under the involution. Thus $\tilde{F}^{\prime}$ is the blowup of $D \times D$ at the points $(q, q),(q, r),(r, q),(r, r)$ and at a further $2 m$ points.

Set $c=\epsilon_{m}\left(D^{(2)},(a+b) x-(\delta / 2)\right)$ and consider the $\mathbb{R}$-divisor

$$
H^{\prime}=\pi^{*} H-c E^{\prime}
$$

where $H$ is the divisor defined in (3.4). Then exactly as in the proof of Theorem 1.2, $\left.H^{\prime}\right|_{E}$ is nef and the hypothesis on the Seshadri constant and Lemma 2.6 imply that the pullback of $\left.H^{\prime}\right|_{F^{\prime}}$ to $\tilde{F}^{\prime}$ is also nef. Thus $\left.H^{\prime}\right|_{\mathcal{Y}_{t}^{\prime}}$ is nef for very general $t$ and using (2.6) once again proves the theorem.

Proof of Corollary 1.7. Fix $g \geq 1$. Let $h$ be the class of the hyperplane in $\mathbb{P}^{2}$ and set $b=\epsilon_{g}\left(\mathbb{P}^{2}, h\right)$. The second symmetric product of a genus 0 curve is $\mathbb{P}^{2}$ and under this identification $x=(\delta / 2)=h$. We have

$$
\epsilon_{g}\left(\left(\mathbb{P}^{1}\right)^{(2)},(1+b) x-b(\delta / 2)\right)=\epsilon_{g}\left(\mathbb{P}^{2}, h\right)=b .
$$

Thus repeated use of Theorem 1.6 yields for a very general curve $D$ of genus $g-1$

$$
\epsilon_{1}\left(C^{(2)},(1+b) x-b(\delta / 2)\right) \geq b,
$$

and so the result follows from Theorem 1.2.

\section{Application to the case $g=5$}

We now prove that if $C$ is a very general curve of genus 5 then $\tau(C) \leq 16 / 7$. This is done by estimating the Seshadri constant at a very general point $p$ of $D^{(2)}$ where $D$ is a very general curve of genus 4 . By $(2.2)$ we know that $\tau(D)=2$. Set $a=16, b=7$ and $L=(a+b) x-b(\delta / 2) \in N^{1}\left(D^{(2)}\right)$ which is ample. By (1.2) it is sufficient to show the following

Claim: If $p$ is a very general point in $D^{(2)}$ then

$$
\epsilon\left(p ; D^{(2)}, L\right) \geq b=7 .
$$

The proof of the claim will use the ideas of Ein-Lazarsfeld [6]. Rather than using the main result of that paper we get an improvement by using their techniques and special properties of the particular surface $D^{(2)}$. In particular we will need the following lemma.

Lemma 4.2 (Ein-Lazarsfeld [6]). Let $X$ be a smooth surface and $L$ be an integral ample line bundle (or class) on $X$. Suppose $\left\{p_{t} \in E_{t}\right\}_{t \in T}$ is a one-parameter family consisting of a point $p_{t}$ in a curve $E_{t} \subset X$ such that mult $_{p_{t}} E_{t} \geq m$ for all $t$. Suppose in addition that $E=E_{0}$ is reduced and irreducible and moreover that the KodairaSpencer class of this family is non-zero. Then $E^{2} \geq m(m-1)$. 
Now suppose $E \subset D^{(2)}$ is a reduced irreducible curve passing through a very general point $p$ with numerical class $(n+\gamma) x-\gamma(\delta / 2)$.

Claim: We have L.E $\geq 7$ and if $(n, \gamma) \notin\{(1,0),(3,1),(5,2)\}$ then L.E $\geq 4 b=28$.

To see this note that $L . E=a n-4 b \gamma=16 n-28 \gamma$ so certainly if $\gamma \leq 0$ then $L . E \geq 28$ unless $(n, \gamma)=(1,0)$. So suppose that $\gamma>0$. As $\tau(D)=2$ we must have $n \geq 2 \gamma$ with equality if and only if $E$ has zero self intersection.

Now for each fixed $\gamma \geq 0$ there are at most finitely many irreducible curves $E$ with numerical class $(n+\gamma) x-\gamma(\delta / 2)$ and self-intersection zero. Since $p$ is assumed to be very general we may assume there is no irreducible curve of zero self-intersection through $p$, and so we in fact have $n \geq 2 \gamma+1$. Then it is easily checked that L.E $\geq 7$ and $L . E \geq 28$ except when $(n, \gamma) \in\{(3,1),(5,2)\}$.

We now finish the proof of (4.1). Suppose for contradiction that $\epsilon\left(p ; D^{(2)}, L\right)<7$ for a very general $p \in D^{(2)}$. Then through a very general point $p$ there exists a reduced irreducible curve $E$ with $m=$ mult $_{p} E$ and

$$
\frac{L . E}{m}<b=7 .
$$

As in [6] the collection of pairs $(p, E)$ consisting of a point $p$ in an irreducible curve $E$ such that $\operatorname{mult}_{p}(E)>L . E / 7$ consists of a countable collection of algebraic families and the proof will be completed by showing that any such family with $p$ a very general point is discrete.

To this end suppose for contradiction that there is a family $\left\{p_{t} \in E_{t}\right\}_{t \in T}$ with $E_{t}$ reduced and irreducible and mult $_{p_{t}} E_{t}>L . E_{t} / 7$ for all $t$. Set $(p, E)=\left(p_{0}, E_{0}\right)$. Since $p$ is assumed to be very general we have from the above that $L . E_{0} \geq 7$ so mult $p_{p_{t}}>1$. Since mult $E_{t}=1$ for a general point $y$ of $E_{t}$ we deduce that the curves $E_{t}$ are moving in a non-trivial family and thus from (4.2) we have that $E^{2} \geq m(m-1)$.

Case 1: $(n, \gamma)=(1,0)$. If $E$ is not the irreducible curve $x_{p}=\{p+q \mid q \in C\}$ then $m \leq E . x=1$ so $m=1$ which is impossible by (4.3). On the other hand if $E=x_{p}$ then $m=1$ and $L . E=a$ which again is absurd.

Case 2: $(n, \gamma)=(3,1)($ resp. $(n, \gamma)=(5,2))$. As $m(m-1) \leq E^{2}=5$ (resp. $m(m-1) \leq$ $E^{2}=9$ ) we have $m \leq 2$ (resp. $m \leq 3$ ). But this implies $\frac{L . E}{m} \geq \frac{3 a-4 b}{2}=10 \geq b$ (resp. $\frac{L . E}{m} \geq \frac{5 a-8 b}{3}=8 \geq b$ ) which in both cases is impossible by (4.3).

Case 3: $(n, \gamma) \neq(1,0),(3,1),(5,2)$. Here we follow [6] but first note that by the previous claim in this case $L . E \geq 4 b$ which by (4.3) implies that $m \geq 5$. Again from (4.3) we have $L . E<7 m$ so $L . E \leq 7 m-1$. Thus by the Hodge index theorem

$$
m(m-1) \leq E^{2} \leq \frac{(L \cdot E)^{2}}{L^{2}} \leq \frac{(7 m-1)^{2}}{60}
$$

which is impossible for $m \geq 5$. 


\section{Acknowledgements}

I would like to thank Robert Lazarsfeld for helpful discussions and for pointing out that the hope that the analogy of Conjecture 1.3 holds for all surfaces was too optimistic. I also thank Ciro Ciliberto, Johan de Jong, Ian Morrison and Yusuf Mustopa.

\section{References}

[1] E. Arbarello, M. Cornalba, P. Griffiths, and J. Harris, Geometry of algebraic curves. Vol. I, Grundlehren der Mathematischen Wissenschaften [Fundamental Principles of Mathematical Sciences], 267 Springer-Verlag, New York, (1985)

[2] T. Bauer, Seshadri constants on algebraic surfaces, Math. Ann. 313 (1999), no. 3, 547-583.

[3] F. Campana and T. Peternell, Algebraicity of the ample cone of projective varieties, J. Reine Angew. Math. 407 (1990) 160-166.

[4] C. Ciliberto and A. Kouvidakis, On the symmetric product of a curve with general moduli, Geom. Dedicata 78 (1999), no. 3, 327-343.

[5] L. Ein, O. Küchle, and R. Lazarsfeld, Local positivity of ample line bundles, J. Differential Geom. 42 (1995), no. 2, 193-219.

[6] L. Ein and R. Lazarsfeld, Seshadri constants on smooth surfaces, Astérisque 218 (1993) 177186.

[7] B. Harbourne and J. Roé, Multipoint Seshadri constants on $\mathbb{P}^{2}$, Rend. Sem. Mat. Univ. Politec. Torino 63 (2005), no. 1, 99-102.

[8] A. Kouvidakis, Divisors on symmetric products of curves, Trans. Amer. Math. Soc. 337 (1993), no. $1,117-128$.

[9] - On some results of Morita and their application to questions of ampleness, Math. Z. 241 (2002), no. 1, 17-33.

[10] R. Lazarsfeld, Positivity in algebraic geometry. I., Ergebnisse der Mathematik und ihrer Grenzgebiete. 3. Folge, Springer-Verlag, Berlin (2004)

[11] K. Oguiso, Seshadri constants in a family of surfaces, Math. Ann. 323 (2002), no. 4, 625-631.

[12] G. Pacienza and F. Polizzi, On a degeneration of the symmetric product of a curve with general moduli, Matematiche (Catania) 56 (2001), no. 2, 297-307 (2003). PRAGMATIC, 2001 (Catania).

[13] B. Strycharz-Szemberg and T. Szemberg, Remarks on the Nagata conjecture, Serdica Math. J. 30 (2004), no. 2-3, 405-430.

[14] T. Szemberg and H. Tutaj-Gasińska, General blow-ups of the projective plane, Proc. Amer. Math. Soc. 130 (2002), no. 9, 2515-2524 (electronic).

[15] G. Xu, Curves in $\mathbf{P}^{2}$ and symplectic packings, Math. Ann. 299 (1994), no. 4, 609-613.

Department of Mathematics, Columbia University, New York, NY 10027. USA.

E-mail address: jaross@math.columbia.edu 